\title{
Modelos de negocio ajustados para proyectos periodísticos reposados: subsistencia económica de medios slow
}

\section{Tight business models for slow journalism projects: economic subsistence of slow media outlets}

\author{
Antxoka Agirre-Maiora; Imanol Murua-Uria; Beatriz Zabalondo-Loidi
}

Cómo citar este artículo:

Agirre-Maiora, Antxoka; Murua-Uria, Imanol; Zabalondo-Loidi, Beatriz (2020). "Modelos de negocio ajustados para proyectos periodísticos reposados: subsistencia económica de medios slow". Profesional de la información, v. 29, n. 6, e290620.

https://doi.org/10.3145/epi.2020.nov.20

Artículo recibido el 25-02-2020 Aceptación definitiva: 23-04-2020
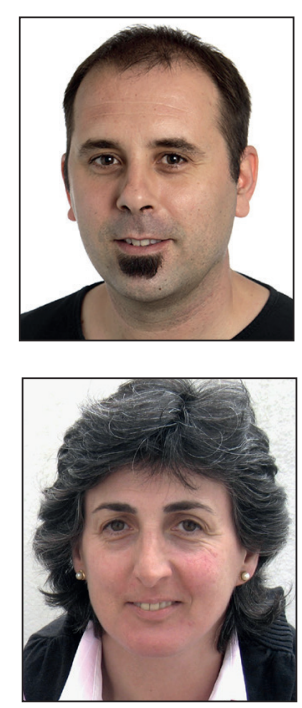

Antxoka Agirre-Maiora $\bowtie$ https://orcid.org/0000-0003-0470-936X

Universidad del País Vasco/Euskal Herriko Unibertsitatea Departamento Periodismo

Barrio Sarriena, $\mathrm{s} / \mathrm{n}$. 48940 Leioa (Bizkaia), España antxoka.agirre@ehu.eus

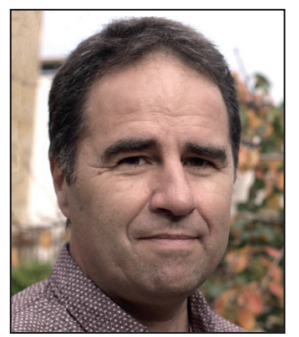

Imanol Murua-Uria https://orcid.org/0000-0003-1071-7446

Universidad del País Vasco/Euskal Herriko Unibertsitatea

Departamento Periodismo

Barrio Sarriena, $\mathrm{s} / \mathrm{n}$.

48940 Leioa (Bizkaia), España

imanol.murua@ehu.eus

\section{Resumen}

En un contexto mediático digital donde se impone la brevedad, instantaneidad y superficialidad de las informaciones, medios digitales comprometidos con el periodismo reposado (slow journalism) apuestan por la calidad, la profundidad y la credibilidad de sus contenidos. Es un modelo de periodismo que, desde los nuevos soportes digitales, revaloriza géneros del periodismo clásico como reportajes, crónicas y entrevistas de largo recorrido y análisis en profundidad. Inmersos en un océano de contenidos digitales gratuitos, estos medios se resienten de la crisis que afecta a las empresas informativas. Este artículo analiza los modelos de negocio que han desarrollado cinco medios digitales españoles que apuestan por el periodismo reposado: 5W, CTXT, Jot down, Panenka y Yorokobu. El gran reto de este tipo de medios es hacer frente a las dificultades para rentabilizar sus audiencias digitales. La metodología utilizada en esta investigación combina estudios de caso de los medios analizados, una encuesta Delphi a profesionales y expertos en la materia, y una encuesta de opinión a consumidores de prensa digital. Del resultado de la investigación se deduce que estos medios sobreviven

\section{Financiación}

Este artículo es producto del proyecto de investigación “Cartografía del periodismo reposado/lento (slow journalism) digital en español: Recopilatorio de prácticas innovadoras y de calidad", referencia 2016-76020-R (AEI, Feder, CE), cofinanciado por el Ministerio de Economía y Competitividad, la Agencia Estatal de Investigación, el Fondo Europeo de Desarrollo Regional y la Comisión Europea. 
gracias a modelos empresariales muy ajustados que no acaban de afianzarse y que limitan el desarrollo de sus proyectos periodísticos. Así mismo, se concluye que los ingresos por suscripciones y las aportaciones voluntarias tienen gran peso en la facturación, y están muy atentos a la fidelización de la comunidad de lectores. También recurren a la diversificación para equilibrar su balance económico con la venta de publicaciones propias en papel o produciendo contenidos para otras empresas. Los proyectos nativos digitales estudiados se sustentan en plantillas reducidas complementadas con el trabajo de amplias redes de colaboradores, en su mayoría periodistas freelance.

\title{
Palabras clave
}

Periodismo; Periodismo reposado; Periodismo slow; Periodismo digital; Prensa digital; Medios slow; Proyectos periodísticos; Modelos de negocio; Subsistencia económica; Medios digitales; Cibermedios; 5W; CTXT; Jot down; Panenka; Yorokobu.

\begin{abstract}
Within the digital media context which imposes brevity, immediacy, and superficiality of information, digital media outlets committed to slow journalism are opting for quality, depth, and credibility in their content. This is a journalistic model which, from new digital platforms, reappraises classical journalism genres such as reports, features, and extensive interviews and in-depth analysis. Immersed in a sea of free digital content, these media outlets are resisting the crisis affecting news companies. This article analyzes the business models developed by five Spanish media outlets committed to slow journalism: 5W, CTXT, Jot down, Panenka, and Yorokobu. The main challenge for this type of media lies in facing the difficulties of capitalizing on their digital audiences. The methodology used in this research combines case studies of the analyzed media, a Delphi survey of professionals and experts in the subject, and an opinion poll of digital press consumers. From the results of our research, we can conclude that these media outlets survive thanks to very tight business models which are struggling to take root and which limit the development of their journalistic projects. Similarly, we conclude that income from subscriptions and voluntary donations represents a significant proportion of their turnover, and that they are very mindful of the loyalty of the readership community. They also resort to business diversification strategies to balance their budgets through the sale of their own paper publications or by producing content for other companies. The native digital projects studied are based on a reduced staff complemented with work by a wide network of collaborators, mostly freelance journalists.
\end{abstract}

\section{Keywords}

Journalism; Slow journalism; Digital journalism; Media outlets; Digital press; Journalism projects; Business models; Economic subsistence; Digital media; Cybermedia; 5W; CTXT; Jot down; Panenka; Yorokobu.

\section{Introducción y estado de la cuestión}

El periodismo slow, entendido como práctica informativa de cocción lenta y de largo formato, surge como reacción al fast journalism, donde priman la rapidez, la novedad, la instantaneidad, la brevedad y la cantidad. El periodismo slow, en cambio, prioriza la calidad frente a la cantidad, huye de la dependencia de la actualización continua a través de una temporalidad más pausada, tanto en la producción como en el consumo, y se decanta por el periodismo de largo formato (longform journalism) con géneros como el reportaje en profundidad, la crónica de corresponsal o la entrevista (Greenberg, 2007; Rauch, 2011; Neveu, 2014; Le-Masurier, 2015; Rosique-Cedillo; Barranquero-Carretero, 2015).

No se trata de un fenómeno nuevo. A lo largo de la historia, tecnologías nuevas han ido implantándose en el proceso de producción informativa, siempre con el afán de alcanzar un seguimiento más rápido y eficaz de la actualidad; pero el periodismo más pausado y de largo formato nunca cayó en desuso (Rosique-Cedillo; Barranquero-Carretero, 2015). Dentro de estas corrientes de periodismo lento destacan dos grandes tendencias:

- el periodismo de investigación, que busca el análisis y la clarificación de las causas de los hechos de los que informa, y

- el periodismo narrativo o literario, que combina técnicas literarias y periodísticas en la elaboración de sus informaciones (Spark, 2012).

La novedad del periodismo slow o pausado del siglo XXI estriba en que surge como reacción a la cada vez mayor obsesión por la rapidez y el consecuente deterioro de la calidad y credibilidad del periodismo dominante actual. La crisis económica y financiera global que azotó a los medios tradicionales condujo a éstos a rentabilizar sobremanera esfuerzos y recursos; muchos se centraron en buscar audiencias digitales y a priorizar la actualización continua e inmediata de contenidos cada vez más breves (Rosenberg; Feldman, 2008; Juntunen, 2010; Saltzis, 2012). Esta tendencia dominante por lo instantáneo y breve ha repercutido notablemente en la calidad de los productos periodísticos: el nivel de superficialidad, simplificación y descontextualización ha aumentado. Como consecuencia, la credibilidad de los medios ha disminuido, tal y como han demostrado diversas investigaciones (Gómez-Mompart; Gutiérrez-Lozano; Palau-Sampio, 2015; Ramírez-de-la-Piscina et al., 2015).

No es posible establecer el momento fundacional del periodismo slow ni de los medios slow, ya que el periodismo reposado y los medios especializados en ella existen desde mucho antes de que Greenberg (2007) acuñara por primera 
vez el término slow journalism y Rauch (2011) el de slow media. La filosofía de estos medios se encuadra dentro del movimiento slow global, que tomó cuerpo primero con el slow food (principios de los 80), y que después se extendió a otros ámbitos: el urbanismo (slow cities), la educación (slow schooling) o el trabajo (slow working), entre otros (Rauch, 2011; Honoré, 2012; Rosique-Cedillo, 2012). Si englobamos el quehacer de estos medios digitales slow como reacción a la obsesión por la rapidez y la brevedad en el periodismo de la era digital, podemos apuntar que el periodismo slow surge bien avanzada la primera década del siglo XXI.

Los medios que en la era digital responden a la filosofía slow nacen (o renacen) en el contexto de una crisis global del modelo de negocio de las empresas informativas, en general, y la de la prensa escrita, en particular. Esta última ha experimentado un descenso continuo de las ventas de ejemplares, a la vez que una disminución notable de la inversión publicitaria (APM, 2019).

Aunque específicamente no se refería a las publicaciones slow, Campos-Freire (2010) recordaba que la crisis había obligado a revisar los modelos de negocio de las empresas de comunicación, sobre todo en su búsqueda de ingresos.

El paulatino aumento de la publicidad digital no ha compensado el descenso de ingresos provocado por la caída de la facturación por publicidad en los formatos impresos, ni se espera que llegue a hacerlo a medio plazo. Tampoco las diversas fórmulas de pago por contenidos digitales llegan a acercarse a los niveles de rentabilidad que la venta de prensa en papel producía en épocas anteriores a la crisis de la segunda mitad de la década del 2000 (Casero-Ripollés, 2010; Cea-Esteruelas, 2013; Rojas-Torrijos; Marín-Sanchiz, 2016; Palau-Sampio; Cuartero-Naranjo, 2018).

Los medios digitales nativos, por su parte, siguen experimentando en la búsqueda de modelos de negocio más adecuados, combinando la publicidad digital y el pago por contenido con otras fórmulas innovadoras habitualmente apoyadas en la fidelización de sus consumidores (Briggs, 2012; Vara-Miguel; Díaz-Espina, 2012; Yuste; Cabrera, 2014; Manfredi-Sánchez; Rojas-Torrijos; Herranz-de-la-Casa, 2015; Newman, 2016). Los modelos de negocio de los medios digitales son diversos y las fuentes de ingresos variadas, pero, según diversas investigaciones realizadas en distintos ámbitos geográficos, la publicidad sigue siendo la piedra angular de la financiación de la mayoría de los medios digitales (Wirtz, 2011; García-Avilés; González-Esteban, 2012; Cea-Esteruelas, 2013). Otras investigaciones dan cuenta de publicaciones de periodismo narrativo en español que han optado por un modelo mixto que combina la producción digital con la producción en papel (García-Galindo; Cuartero-Naranjo, 2015).

Las fuentes de financiación de los medios slow no parecen responder a un patrón común, pero, según los incipientes estudios relativos a la parte empresarial y económica de las empresas informativas especializadas en el periodismo reposado, tienen en común que sus modelos de negocio tienen un carácter alternativo bien diferenciado de los criterios eminentemente comerciales de los medios tradicionales (Rodríguez-Rodríguez; Albalad-Aiguabella, 2012; Rosique-Cedillo; Barranquero-Carretero, 2015; Dowling, 2016; Sabaté-Gauxachs; Micó-Sanz; Díez-Bosch, 2019). Tras analizar los modelos de negocio de cuatro medios slow (De correspondent, Delayed gratification, Narratively y The big roundtable), Dowling (2016) argumenta que, más allá de las especificidades de cada cual, todos ellos se alejan de los criterios puramente comerciales, buscan alternativas que dependan lo menos posible de los ingresos por publicidad y confían en la disposición de sus lectores para pagar. Tampoco las fórmulas de pago son homogéneas; las más recurrentes son la suscripción, los micro-pagos por contenidos determinados, las donaciones o iniciativas de crowdfunding, e incluso, más de una a la vez, a cambio de un producto periodístico cuyo valor añadido deriva de su elaboración artesanal.

Algunos autores que mencionan la economía de los medios slow coinciden en destacar el escaso peso relativo de los ingresos por publicidad en sus cuentas, la existencia de un nicho de mercado de consumidores dispuestos a pagar por los contenidos (Greenberg, 2012; Drok; Hermans, 2016) y el creciente peso de fórmulas innovadoras basadas en la fidelidad de sus lectores (Casero-Ripollés, 2010; Rodríguez; Albalad, 2012; Rosique-Cedillo; Barranquero-Carretero, 2015). Estos autores también coinciden en señalar que la mayoría de los medios slow viven en una incertidumbre económica al no lograr afianzar un modelo de negocio estable y seguro y, en gran medida, basan su viabilidad económica en una estructura reducida de bajos costes (Palau-Sampio; Cuartero-Naranjo, 2018).

La filosofía slow va a contracorriente de los criterios eminentemente comerciales de los medios tradicionales, como se puede apreciar claramente en los principios del Slow media manifesto propuesto por los blogueros alemanes Köhler, David y Blumtritt (2010). En este manifiesto reivindican, efectivamente, la sostenibilidad tanto en la producción como en el consumo, los altos estándares de calidad por encima de los criterios productivistas y la centralidad de sus comunidades de lectores. Dicho en palabras de Neveu (2016, p. 452):

"Slow journalism transforms its audiences into partners".

El manifiesto hace también referencia al peso secundario que propugnan para la publicidad comercial:

"El éxito de los medios slow no se basa en la abrumadora presión publicitaria en todos los canales".

En definitiva, como destaca Dowling (2016), la filosofía slow tiene implicaciones no solo en el modo de elaborar los contenidos periodísticos de estos medios, sino también en la manera de comercializar y rentabilizar sus productos (Dowling, 2016; Sabaté-Gauxachs; Micó-Sanz; Díez-Bosch, 2019). 


\section{Objetivos y metodología}

El objetivo de esta investigación es avanzar en el conocimiento de los modelos de negocio de medios especializados en el periodismo slow digital en español y detectar cuáles pueden ser las claves para su viabilidad económica. Para responder a este objetivo se han formulado las siguientes preguntas de investigación:

RQ1: ¿Qué tipos de empresas editan los medios analizados y cuáles son sus características principales?

RQ2: ¿Cuáles son las fuentes principales de ingresos de las empresas analizadas?

RQ3: ¿Qué evolución han experimentado sus resultados económicos?

RQ4: ¿Qué grado de similitud presentan los modelos de negocio de los medios slow?

RQ5: ¿Qué grado de innovación presentan los modelos de negocio de los medios slow con respecto a los modelos de los medios convencionales?

RQ6: ¿Cuáles pueden ser las claves para la viabilidad económica y empresarial de los medios slow?

La investigación comenzó en 2017. En la primera fase se realizó una amplia revisión bibliográfica que ayudara a establecer los criterios para identificar diez medios referenciales del slow journalism digital practicado en español. La primera selección contempló treinta portales de periodismo reposado, que se analizaron uno a uno para determinar si cumplían las características que se habían extraído del estudio bibliográfico. Además de seleccionar medios que cumplieran estas expectativas, el equipo de investigación valoró el hecho de que la muestra reflejara un equilibrio geográfico y temático. De este modo se obtuvieron los diez medios que han sido objeto de estudio: Jot down (España), Yorokobu (España), $5 W$ (España), CTXT (España), Panenka (España), La silla vacía (Colombia), Arcadia (Colombia), Anfibia (Argentina), Letras libres (México) y Gatopardo (México).

A continuación se trabajó en un estudio de caso por cada medio seleccionado. Yin (1992) define el estudio de caso como una indagación empírica que utiliza múltiples fuentes de conocimiento para investigar un fenómeno actual dentro de un contexto de vida real. Además de las fuentes previas, otras nuevas completaron la documentación de cada medio; la observación directa permitió contextualizar cada medio e identificar variables comunes y/o específicos entre ellos. Por último la información recogida fue completada y contrastada mediante entrevistas en profundidad (Wimmer; Dominick, 1996) realizadas a los responsables periodísticos, económicos y tecnológicos de cada publicación (entre marzo y julio de 2018). En total se realizaron 26 entrevistas de forma presencial, excepto la de Anfibia (Argentina) que se realizó por videoconferencia.

Otro de los métodos cualitativos utilizados en esta investigación ha sido un estudio Delphi internacional. Este es un método de naturaleza prospectiva: busca obtener una visión consensuada de expertos sobre un tema determinado (Landeta, 1999; Linstone; Turoff, 2002; Manias-Muñoz; Jin; Reber, 2019; Kent; Saffer, 2014). En nuestro caso se buscó un plantel de expertos que fueran académicos y/o profesionales, se cuidó la paridad de género entre ellos y también la diversidad de su procedencia. En la primera oleada, realizada entre mayo y julio del 2018, respondieron 28 participantes. Las preguntas se constituyeron en torno a diez bloques: 'Estado del periodismo', 'Función del periodismo narrativo de calidad', 'Características del periodismo narrativo de calidad', 'Géneros del periodismo narrativo de calidad', 'Perfil del emisor', 'Perfil del lector-a', 'Viabilidad económica', 'Estructura empresarial', 'Futuro del periodismo impreso' y 'Futuro del periodismo narrativo de calidad'. En la segunda ronda, realizada entre marzo y junio de 2019 , se incluyeron las mismas preguntas de la primera, pero cada pregunta iba acompañada de los resultados centrales de la primera ronda, combinada con una escala de Likert. Gracias a este sistema se evaluó y cuantificó el grado de conformidad o disconformidad que señalaban los expertos en sus respuestas, así como alguna posible variación o matización. Fueron 25 los participantes que respondieron en la segunda ronda: el $48 \%$ fueron mujeres, y el $52 \%$, hombres. Procedían de América del Norte, América del Sur, Australia y Europa. El número de expertos académicos $(53,78 \%)$ fue algo superior al de profesionales $(39,29 \%)$. El resto $(7,14 \%)$ se autodefinió como académico y profesional a la vez. Durante todo el proceso se garantizó el anonimato de los expertos.

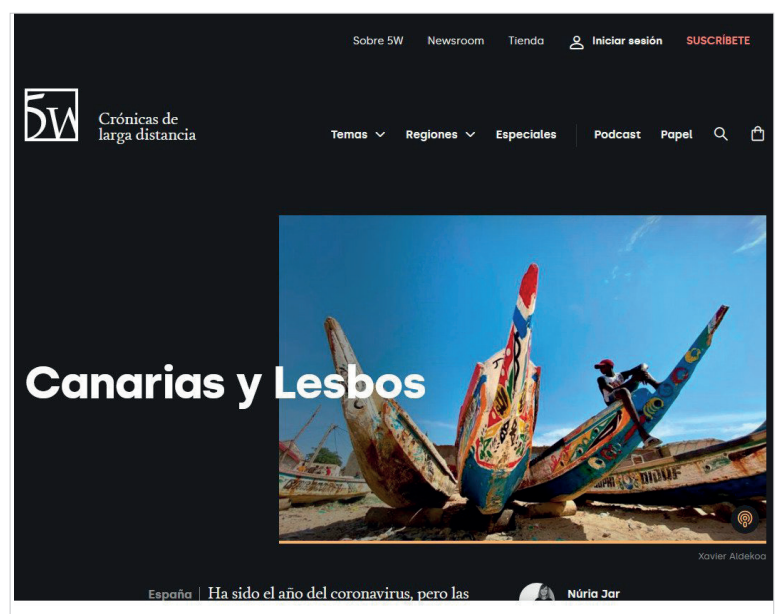

Esta web, como todas, usa cookies. Sl estás de acuerdo, pincha en 'Aceptar. Más Información Aceptar
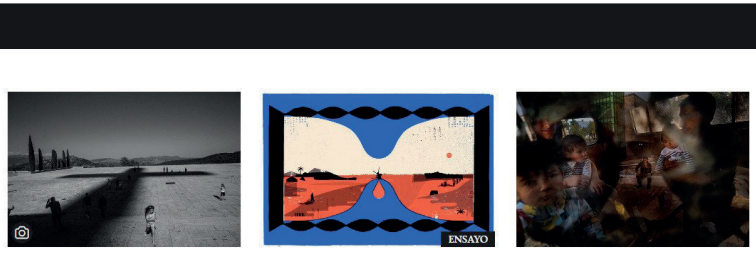

Hijos de la desmemoria Paterna, España | Centenares de personas Ilevan décadas tratando de
localizar a sus farmiliares asesinados durante la dictadura franquist sonti Donaire

El peso del silencio Mi ciudad, $\mathrm{mi}$ sáhara | Esto solo puedo contarlo en
primera persona: el fracaso de la paz tumba el triunfo del olvido en el Sáhara Ebbaba Hameic Stepanakert, Nagorno-Karabaj| Las Stepanakert, la capital de Nagor Karabaj Karlos Zurutuza | Ricard Garcia Vilanova

https://www.revista5w.com 
Completó la investigación una encuesta dirigida a lectores de prensa digital (personas entre 18 y 65 años) residentes en España, Argentina, Colombia y México. Las entrevistas las realizó online la empresa CIES S. L., teniendo en cuenta cuotas de sexo y edad. Se preparó un cuestionario de 40 preguntas cualitativas y cuantitativas: una parte de ellas se refería a la opinión y conocimiento sobre el periodismo reposado, y la otra, a los hábitos de consumo. La muestra comprendió 2.000 encuestas (500 por país), con un nivel de confianza del 95\% y un margen de error máximo del 2,2\% para resultados globales. Se realizó del 16 al 23 de julio de 2019.

De los diez medios analizados en el proyecto de investigación, este artículo se detiene en los cinco que se editan en España: 5W, CTXT, Jot down, Panenka y Yorokobu. Se han comparado sus estructuras empresariales y modelos de negocio, ya que están regulados por un mismo marco jurídico y buscan su viabilidad económica en un contexto socioeconómico y mediático similar.

Tabla 1. Características empresariales y entrevistas realizadas

\begin{tabular}{|l|l|l|l|l|l|}
\hline Revista & Fundación & Editora & Sede & Temática & Entrevistas realizadas \\
\hline Yorokobu & 2009 & Brands \& Roses S.L. & Madrid & $\begin{array}{l}\text { Innovación, } \\
\text { creatividad, } \\
\text { tendencias }\end{array}$ & $\begin{array}{l}\text { Mar Abad (jefa de redacción), Marcus Hurst (jefe de } \\
\text { redacción y responsable de web y redes sociales); } \\
\text { Fermin Abella (director comercial) y Juanjo Moreno } \\
\text { (director). Todos, socios fundadores. }\end{array}$ \\
\hline Jot down & 2011 & Wabi Sabi Investments S.L. & Sevilla & Cultura & $\begin{array}{l}\text { Ángel Luis Fernández, consejero delegado, editor y } \\
\text { uno de los socios fundadores. }\end{array}$ \\
\hline Panenka & 2011 & Panenka S.L. & Barcelona & Fútbol & $\begin{array}{l}\text { Roger Xuriach (coordinador); Àlex López-Vendrell } \\
\text { (gerente); Carlos Martín-Río (redactor jefe); Marcel } \\
\text { Beltrán (redactor jefe, director de la web y responsa- } \\
\text { ble de las redes sociales). }\end{array}$ \\
\hline CTXT & 2015 & Revista Contexto S.L. & Madrid & Generalista & $\begin{array}{l}\text { Miguel Mora (director) y Vanesa Jiménez (directora } \\
\text { adjunta). Ambos, socios fundadores. }\end{array}$ \\
\hline 5W & 2015 & Colectivo 5W S.L. & Barcelona & $\begin{array}{l}\text { Información } \\
\text { internacional }\end{array}$ & $\begin{array}{l}\text { Agus Morales (director), Maribel Izcue (responsa- } \\
\text { ble área económica), Marta Arias (redes sociales y } \\
\text { publicidad). }\end{array}$ \\
\hline
\end{tabular}

Fuente: $H G H$

\section{Resultados}

\subsection{Emprendimiento en plena crisis}

Yorokobu comenzó su andadura en 2009; Jot down y Panenka lo hicieron en 2011, y tanto CTXT como 5W, en 2015. Son años en los que la crisis del modelo tradicional de negocio basado en las ventas del papel, que arranca a mitad de la década de 2000, se ve agravada a partir de 2008 por la crisis económica global: en España, de 2008 a 2013 se cerraron 375 medios de comunicación, y 12.200 periodistas perdieron su puesto de trabajo (APM, 2015).

Algunos de estos proyectos los encabezan profesionales que han dejado de trabajar en grandes empresas de comunicación a causa de la situación generada por la crisis. En el acto de presentación en sociedad de CTXT, en octubre de 2015, el periodista Jordi Évole dijo que aquello parecía "una reunión de extrabajadores de El país" (Mora, 2018). Los fundadores de $5 W$, en su mayoría fotoperiodistas que venían de trabajar la información internacional sobre el terreno, cuentan que decidieron poner en marcha el proyecto porque los grandes medios les obligaban a malvender su trabajo (Morales, 2018).

Los cinco proyectos se podrían encuadrar dentro del denominado "periodismo emprendedor": un conjunto de iniciativas creadas desde 2008 por periodistas para afrontar la transformación del mercado periodístico español, a través de estrategias de autoempleo (Casero-Ripollés; Cullell-March, 2013; Manfredi-Sánchez; Rojas-Torrijos; Herranz-de-la-Casa, 2015). En todos los casos estudiados el emprendimiento responde a la necesidad de articular una oferta que no encuentra cabida en los medios convencionales. Yorokobu lo puso en marcha un grupo jóvenes emprendedores provenientes del mundo de la comunicación y el marketing creando lo que Naudín-Escuder (2018) califica como la revista millennial más importante junto a Vice y PlayGround. La filosofía del do it yourself está recogida en su página de Facebook:

"Aquel año nos dimos cuenta de que no encontrábamos ninguna publicación que tratase los temas sobre los que queríamos leer y decidimos que lo haríamos nosotros mismos".

Jot down nació a través de un foro de internet denominado Areópago, un sitio virtual en el que coincidieron personas muy diversas a las que les unía un diagnóstico común: estaban aburridas de tener que "cargar" con cinco revistas diferentes cada fin de semana para poder encontrar los contenidos que les interesaban (Fernández, 2018). Panenka surgió con la idea de publicar la primera revista de cultura futbolística del país, un producto que ya existía en otros países europeos, como So Foot en Francia o 11 Freunde en Alemania (Martín-Río, 2018). 5W surgió de la constatación de la inexistencia de una publicación consolidada de información internacional de crónicas y fotografía (Morales, 2018). Por último, CTXT buscaba ofrecer en internet las esencias del periodismo clásico, volver "a los géneros clásicos que la prensa en internet había abandonado", publicando largas entrevistas de fondo, grandes reportajes y crónica de corresponsal reposada (Mora, 2018). 
Son proyectos que responden a distintos ámbitos temáticos pero que coinciden en su apuesta por el periodismo pausado en internet, como evidencian sus propias cabeceras: 'Take a walk on the slow side' (Yorokobu), 'Orgullosas de llegar tarde a las últimas noticias' (CTXT), 'El fútbol que se lee' (Panenka), y 'Crónicas de larga distancia' (5W); o, en el caso de Jot down, en el guiño que hace el nombre de la publicación:

"Se trata de una disonancia cognitiva, porque 'jot down' significa 'tomar notas cortas' y nosotros lo que hacemos son artículos largos" (Fernández, 2018).

Esta apuesta por el slow journalism resulta palpable en la extensión de los textos que publican, mucho mayor de lo que suele ser habitual en los medios digitales convencionales. Los textos publicados en Yorokobu tienen habitualmente una extensión de 800 a 2.400 palabras; los de Jot down entre 1.500 y 4.000 palabras, con entrevistas que superan las 10.000 ; los textos de Panenka entre 1.500 y 10.000 ; los de $5 W$ alrededor de 2.500 , y los de CTXT superan las 1.500 palabras. La cantidad de textos publicados, en cambio, es mucho menor que el habitual en los medios convencionales: entre 20 y 50 textos a la semana, en todas las revistas.

\subsection{Sociedades limitadas con espíritu cooperativista}

Los cinco proyectos analizados se constituyeron como sociedades limitadas. La sociedad matriz de Yorokobu es Brands \& Roses S. L., una empresa de contenidos en la que participaron los cuatro socios fundadores con 3.000 euros cada uno. Jot down lo edita Wabi Sabi Investments S. L., creada al 50\% entre su editor y su directora, a quienes se han ido sumando nuevos socios "entre los que hay trabajadores, colaboradores, lectores y amigos" (Fernández, 2018). Panenka S.L. consta de 17 socios, pero el socio principal es Aitor Lagunas, fundador y editor. Colectivo 5W S. L. tuvo nueve socios fundadores con la misma participación en la empresa y han desarrollado el proyecto sin la intervención de ningún otro socio. Por último, Revista Contexto S. L. fue fundada por catorce socios, cada uno de los cuales aportó 1.000 euros. Tras varias ampliaciones de capital, en 2020 la empresa cuenta con 144 socios: alrededor de la mitad son periodistas y colaboradores del medio y la otra mitad lectores.

Aunque los casos estudiados se han decantado por la sociedad limitada como forma jurídica, varios de ellos funcionan más como cooperativas de periodistas. En $5 W$ subrayan que su modelo está muy lejos de "la mentalidad corporativa empresarial" y que el espíritu del equipo "es cooperativo y de grupo" (Izcue, 2018). En Jot down también señalan que funcionan "como una cooperativa" (Fernández, 2018). Los expertos consultados en la encuesta Delphi de esta investigación coinciden también en que las estructuras empresariales que mejor se adaptan a las empresas de periodismo slow son las sociedades limitadas, cooperativas de trabajadores y otros modelos híbridos que combinan el negocio y el non-profit ( $76 \%$ de consenso en la segunda oleada).

Todos los proyectos tienen estructuras de reducidas dimensiones para minimizar gastos. Yorokobu y Jot down no tienen una redacción centralizada, el personal trabaja por teletrabajo. $5 \mathrm{~W}$ tampoco tuvo sede central hasta que en el 2017 lo situaron en Barcelona.

La plantilla de Yorokobu es de 25 trabajadores, once de ellos en redacción (incluida la dirección) y el resto en el área comercial y de administración. En Jot down trabajan nueve personas a jornada completa y cinco a parcial. Panenka cuenta con seis personas a jornada completa y tres a parcial. Así mismo, cuentan con dos estudiantes de periodismo en prácticas; un consejo de redacción, en la que se diseñan los contenidos, y varios redactores, que colaboran como autónomos. En CTXT la plantilla estable del medio se reduce a los tres miembros de su dirección; cuentan también con dos periodistas a media jornada, y tienen varios responsables de distintas áreas, tanto periodísticas como administrativas, que no forman parte de la plantilla. CTXT tiene, además, un consejo editorial que se reúne semanalmente para tratar sobre los contenidos de la revista. En $5 W$ trabajan cinco personas en plantilla, y tienen siete responsables de distintas áreas, que colaboran como autónomos.

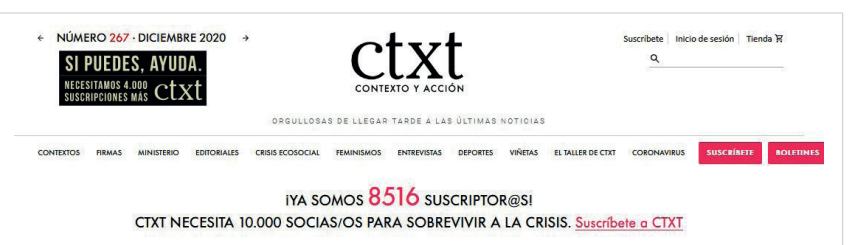

¿Transición modélica o restauración corrupta?

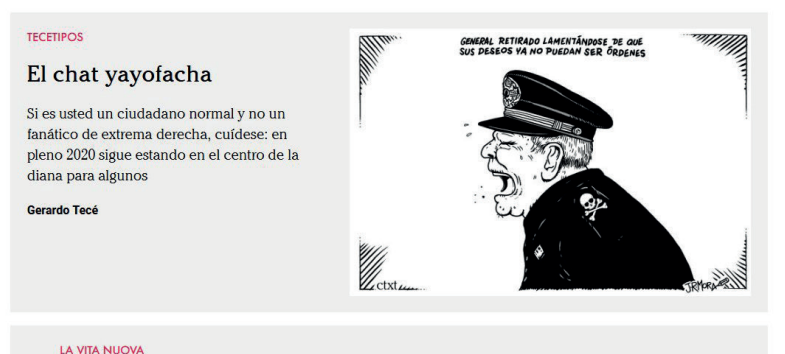

Si le gustó la XXIII promoción le gustará la XIX

El odio es fundamental para una involución. Si el plomo es el material con el que forjan los sueños, el odio lo es para las pesadillas. Odio territorial e ideológico. Perceptible en RR.SS. e información

Guillem Martinez 5 Diviembre 2020
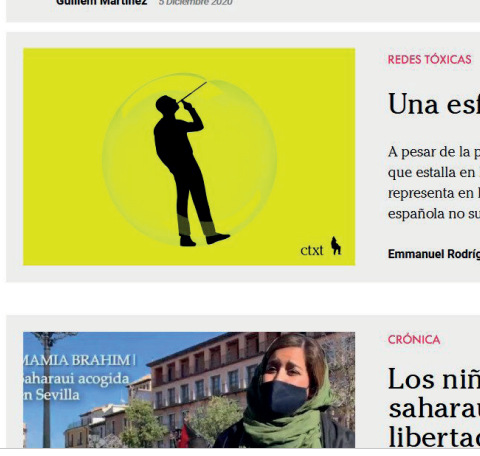

Una esfera pública ¿fallida?

A pesar de la polarización y del aparente guerracivilismo que estalla en las redes y que de vez en cuando se representa en las tertulias de televisión, en la politica española no sucede nada

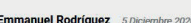

RÓNII A

Los niños de la diáspora saharaui, la voz de la libertad

https://ctxt.es 
Con la salvedad de Yorokobu y de Jot down, que al ser los proyectos que más recorrido han tenido han podido desarrollar un tamaño medio, se trata de plantillas muy reducidas, y en todos los casos gran parte de sus contenidos los producen a través de colaboradores: en la web de Yorokobu aparecen contabilizados 420 autores, en Jot down son varias docenas, en Panenka la nómina de colaboradores habituales ronda las 20-25 firmas, y en CTXT y $5 W$ un centenar en cada uno de ellos.

Además de los gastos salariales del personal de plantilla, los cinco medios analizados disponen de importantes partidas económicas para pagar los numerosos contenidos producidos por colaboradores ajenos a la plantilla. En cuatro casos todos los trabajos son retribuidos, y solamente en Panenka algunos de los contenidos de la web se realizan de forma voluntaria y gratuita. En Yorokobu se pagan 50 euros por cada artículo para la web, y 100 euros por cada artículo de la revista en papel. En Jot down, el mínimo es de 75 euros por un trabajo de 1.200 palabras y se puede llegar a pagar hasta 300 euros por trabajos de mayor extensión. En CTXT la pieza de opinión se paga 60 euros, y los grandes reportajes hasta 120 euros. Los responsables de las publicaciones consideran que estos precios se sitúan dentro de lo que marca el mercado (Mora, 2018).

\subsection{Modelos de negocio ajustados}

La encuesta realizada por CIES en julio de 2019 para nuestra investigación confirma la relevancia que ha adquirido la prensa digital: en España, el $85 \%$ de los encuestados se informa "habitualmente" a través de la prensa digital y el $42 \%$ lo hace de una "forma prioritaria". La encuesta también señala que sólo el $11 \%$ de los encuestados ha pagado por una suscripción de prensa digital y sólo el $27 \%$ se muestra dispuesto a pagar por el consumo de contenidos de calidad. Así mismo, se ha podido detectar un grado importante de desconocimiento de los medios slow: tan sólo el $16 \%$ de los encuestados han oído hablar de los términos "periodismo narrativo", "periodismo lento", "periodismo reposado" o slow journalism, y solamente un $36 \%$ de los encuestados declara haber leído alguna vez prensa narrativa (esto, tras haberles informado de la definición y de algunos ejemplos de marcas). Por otro lado, cabe destacar que la proporción de lectores dispuestos a pagar por contenidos de calidad sube hasta el 54\% entre quienes son lectores de medios slow. Los datos de la encuesta a lectores de prensa digital coinciden en lo sustancial con lo recogido en la encuesta Delphi, que advierte que los medios slow actúan en un mercado de reducidas dimensiones.

Estos medios actúan, por tanto, en un mercado de consumidores de prensa digital donde la gratuidad es regla común y el pago por contenidos sigue siendo minoritario. La disposición a pagar a cambio de calidad es mayor en los lectores de medios slow, pero no deja de ser una pequeña minoría dentro de los lectores de la prensa digital en general. Muestra de ello es que cuatro de los cinco medios analizados en este trabajo ofrecen gratuitamente sus contenidos digitales, combinado en algunos casos con la venta de productos en papel. En Yorokobu argumentan la dificultad de ser competitivos en un área en el que la oferta de información gratuita es inmensa (Abella, 2018), y en CTXT priorizan la vocación de servicio público del periodismo de calidad (Mora, 2018). De los cinco casos únicamente $5 W$ exige el pago por sus contenidos digitales, una cuota anual de 36 euros.

\subsubsection{La comunidad como soporte básico}

Otra coincidencia entre los expertos consultados en la encuesta Delphi es que los modelos de interacción con la audiencia son fundamentales en el desarrollo -también económico- de este tipo de proyectos. El experto número 25 lo resume así:

"Cuidar la comunidad de lectores es clave para la supervivencia".

Algunos expertos del Delphi sitúan las perspectivas de futuro de los medios slow en la financiación basada en las suscripciones.

CTXT ofrece sus contenidos gratuitamente pero no por ello renuncia a financiarse a través de las suscripciones de sus lectores, a los que propone suscribirse a la revista no para poder leer sus contenidos, sino como aportación voluntaria: 
se impuso en sus inicios el objetivo de llegar a la cifra de 10.000 suscripciones y a finales de 2020 había superado los 8.500. Para conseguir estas suscripciones apelan a la necesidad de este tipo de financiación para garantizar un periodismo independiente:

"La filosofía es que sientan que gracias a su apoyo se puede publicar esto" (Mora, 2018).

Al final de todos los artículos el lector se encuentra con una invitación a suscribirse para que CTXT "siga informando libremente". El director de CTXT considera que los lectores son conscientes de "lo peligroso" que es no pagar por la prensa, porque, si no la pagan ellos, la está pagando alguien, "en este caso los bancos y las grandes empresas" (Mora, 2018). Casi tres de cada cuatro participantes del Delphi incidieron en que es la independencia editorial de estos medios la que conduce o conducirá su viabilidad económica.

En $5 W$ también apelan al apoyo de su comunidad de lectores como base de viabilidad económica, pero a través del clásico modelo de la venta de sus contenidos. Sus responsables hacen hincapié en la necesidad de concienciar al público de que el periodismo independiente y de calidad no puede ser gratuito (Arias, 2018). A principios de 2020, 5W contaba con 3.100 suscriptores y se había propuesto llegar a los 4.000 .

En sus inicios, $5 W$ y CTXT también buscaron el apoyo económico de su comunidad a través de otro mecanismo: el crowdfunding. 5W lanzó su primera campaña en mayo del 2015 en la plataforma Verkami, y para septiembre de ese año logró recaudar 50.030 euros, gracias a la contribución de 893 personas. En aquel mismo año, CTXT recaudó 24.000 euros que se destinaron casi íntegramente a los gastos para el diseño y construcción de la web.

El crowdfunding es en sí mismo una herramienta que construye comunidad, esencialmente colaborativa (Taborda, 2016). También se ha utilizado para realizar alguna producción concreta. En 2018, CTXT desarrolló dos campañas para cubrir los gastos de uno de sus reporteros de más éxito, Guillem Martínez, y para renovar la sede de la revista en Madrid; a principios de 2020 tenía otra iniciativa abierta para financiar el documental Billy, de Max Lemcke. Mediante estas campañas llegan a ingresar una media de unos mil euros al mes (Mora, 2018). Por su parte, $5 W$ financió a través de esta fórmula la cobertura de Siria después del Estado Islámico, realizada por el periodista Mikel Ayestaran y el fotoperiodista Guillem Trius en 2018, proyecto para el que se recaudaron 7.000 euros (Izcue, 2018).

La importancia vital que tiene la comunidad tanto en un medio como en el otro se traduce en multitud de iniciativas que buscan reforzar este lazo. $5 \mathrm{~W}$ organiza encuentros entre los lectores y corresponsales a través de redes sociales (Facebook Live o Periscope), presenta sus trabajos, imparte cursos y talleres donde conversan sobre periodismo internacional, realiza encuestas entre sus suscriptores para que puedan valorar el producto y proponer mejoras, o pone a su disposición sus cuentas anualmente. CTXT tiene una zona de contenidos exclusivos para los suscriptores denominada El saloncito: éstos reciben semanalmente una "carta al suscriptor" de una de las firmas de referencia del medio, en la que comentan temas de actualidad de forma más cercana; también organizan talleres, charlas y laboratorios, además de ofrecer sus cuentas financieras en la web.

\subsubsection{Complementariedad de la web con el papel}

Yorokobu, Jot down y Panenka también cuentan con suscriptores, pero no de sus contenidos digitales sino de sus respectivas revistas impresas.

En el caso de Panenka se trata de una revista mensual con una tirada de 8.000 ejemplares que lleva el mismo nombre y cuyas suscripciones y ventas suponen una gran fuente de financiación: la cuota anual de 45 euros de sus 3.000 suscriptores supuso en 2017 el $27 \%$ de sus ingresos, y la venta en quioscos y librerías otro $20 \%$.

La revista trimestral de Jot down tiene 700 suscriptores, que pagan 60 euros anuales, lo que supone el 5\% de sus ingresos. Hasta enero de 2019 su principal ingreso fue el acuerdo firmado con El país mediante el cual el equipo de Jot down publicaba la revista mensual Jot down smart que se distribuía junto al diario madrileño como suplemento cultural. La venta de productos como la revista infantil Jot down kids y cuatro libros al año con el sello Jot down también son importantes fuentes de ingresos (Fernández, 2018).

El modelo de negocio de Yorokobu se sitúa en otras coordenadas: la publicación es parte de la empresa de contenidos Brand \& Roses cuya principal fuente de financiación es la producción de contenidos para varias marcas (46\% de ingresos). La Yorokobu impresa mensual tiene 3.000 suscriptores que pagan una cuota anual de 45 euros; su tirada es de 30.000 ejemplares que en su gran mayoría se reparten gratuitamente gracias a acuerdos comerciales que reportan ingresos económicos. Las tres vías son importantes como fuentes de financiación (Abad, 2018).

En estos casos la web y las redes funcionan como un escaparate, como la principal herramienta para construir la comunidad que luego se traducirá en ventas y suscripciones del papel: 
“Queremos que la gente conozca la revista a partir de lo que hacemos también online” (Beltrán, 2018).

De esta manera lo digital y el papel se complementan:

"Funcionamos como el software freemium, tenemos miles de artículos gratuitos y luego productos de calidad impresos para algunos de nuestros lectores" (Fernández, 2018).

Y en todos ellos se realiza un esfuerzo importante en crear comunidad: Panenka y Yorokobu, por ejemplo, realizan encuestas entre sus lectores para mejorar el producto. Además, este último ofrece anualmente la posibilidad de hacer la ilustración de una portada de la revista y de votar entre las que se presentan. Como señala Marín (2016, p. 46), hay una apuesta por la implicación del lector,

"en el proceso productivo (...) en las opciones a participar una vez publicadas las informaciones, y a través de la creación de comunidad mediante explicaciones constantes sobre el funcionamiento interno y la muestra de transparencia en la financiación".

Tampoco CTXT y $5 W$ han renunciado a publicar en papel. CTXT cuenta desde 2017 con la revista mensual El dobladillo que tiene una tirada de 3.500 ejemplares, así como libros bajo el sello CTXT. Por su parte, $5 W$ publica la revista anual $5 W$ con una tirada de 5.000 ejemplares y la colección Voces $5 W$ con tiradas de entre 1.500 y 2.000 ejemplares. Estos productos impresos son una vía para reforzar sus comunidades y sus ingresos: se les ofrecen a los suscriptores a un precio especial y se venden tanto online como en papel. La suscripción de CTXT puede variar de la cuota anual básica de 50 euros a los 90 euros con todos los productos impresos y la de $5 W$ de los 36 euros anuales de la cuota básica a los 60 , también con todos los productos impresos.

\subsubsection{Publicidad, alianzas y diversificación}

El peso de la publicidad como fuente de ingresos es muy desigual en los cinco casos estudiados. Solamente en Yorokobu los ingresos de publicidad de la web y la revista impresa (220.611 euros en 2018) superan a los ingresos por suscripciones y ventas (167.507), aunque en el conjunto de la empresa Brand \& Roses la publicidad no supone más que el 10,7\% de los ingresos. Para Panenka y Jot down la publicidad es una fuente de financiación muy importante, el $40 \%$ y el 31,2 \% de sus ingresos respectivamente, pero no llega a ser la fuente principal de ingresos. En el caso de CTXT este porcentaje baja hasta el $14 \%$ y en el de $5 W$ hasta el $3 \%$. Estos dos últimos expresan la importancia de defender su independencia ante las posibles injerencias de los anunciantes. En CTXT, por ejemplo, solo aceptan "patrocinadores y anunciantes transparentes y sobrios, que no interfieran en la línea editorial", se niegan a publicar "contenido patrocinado de índole comercial" y aseguran que prefieren cerrar "antes que aceptar publicidad oculta", según aseguran en su web.

Frente a los medios digitales convencionales que a principios de la pasada década se sustentaban principalmente en la publicidad (Wirtz, 2011; García-Avilés; González-Esteban, 2012; Cea-Esteruelas, 2013) los medios estudiados a finales de década han desarrollado otras vías de financiación importantes tal como vaticinaban varios estudios realizados con anterioridad (Casero-Ripollés, 2010; Rodríguez; Albalad, 2012; Rosique-Cedillo; Barranquero-Carretero, 2015).

Una de esas líneas de trabajo alternativas es la de las alianzas con otras publicaciones. Esta alianza se puede materializar en un acuerdo editorial de trueque gratuito de contenidos, como hacen CTXT y Panenka con varios "medios amigos", o en la compraventa de contenidos, tal como hace CTXT con Público. Este medio publica cuatro piezas de CTXT diarias a cambio de un fijo económico, gestiona algunos de sus espacios publicitarios y contabiliza como propio el tráfico de CTXT en las mediciones de OJD Interactiva (Mora, 2018).

Jot down avanzó un paso más allá en septiembre del 2015 al llegar a un acuerdo con El país para la distribución de la revista Jot down smart. Jot down entregaba mensualmente un fichero en formato pdf con los contenidos de la revista y a cambio recibía 25.000 euros al mes y el $60 \%$ de los ingresos por publicidad. El país imprimía la revista, se encargaba de la distribución y recaudaba el $40 \%$ de la publicidad además de lo correspondiente a la venta de la revista en los quioscos, que solía salir el primer domingo de cada mes a 3 euros el ejemplar. Este acuerdo se rompió en enero de 2019 al variar ostensiblemente las condiciones impuestas por El país a Jot down. La ruptura del acuerdo en enero de 2019 supuso un serio contratiempo para la revista ya que suponía perder su principal fuente de ingresos en 2018, un 35,8\% del total (Fernández, 2018).

Jot down ha probado diversas fórmulas de alianzas bastante novedosas. Un ejemplo de ello es la revista impresa Five que publicaron en 2013 junto a otras cuatro publicaciones entre las que se encontraba Yorokobu (se unieron también Diario Kafka, Politikon y Naukas),

"Invitamos a cinco medios digitales a que participaran con una quinta parte del contenido. El producto lo pagamos enteramente nosotros, pero los beneficios se dividieron entre cinco. A la hora de vender ese producto, ellos dieron a conocer Five a su comunidad y esa comunidad desembarcó en Jot down para comprar su producto y a su vez conocer lo que hacíamos" (Fernández, 2018). 
Además de buscar alianzas, los proyectos analizados han optado, en menor o mayor grado, por diversificar su actividad para encontrar nuevas vías de financiación.

El que más ha desarrollado esta vía es Yorokobu, con una empresa matriz, Brand \& Roses, cuya actividad principal es crear contenidos para marcas comerciales. El equipo de Yorokobu ha creado cartelería, blogs, anuncios, ilustraciones, vídeos, microrrelatos, infografías, iconos, etc. para clientes importantes: Coca-Cola, Fundación Telefónica, Desigual, Gobierno de Suiza, Junta de Extremadura, BBVA, Nissan, Heineken, Pacha, Fox Live... La forma de contar y describir las cosas en Yorokobu les ha posibilitado afianzarse en el mercado de contenidos (Abella, 2018). Un ejemplo de ello es Ling, la revista impresa que la aerolínea Vueling ofrece a sus usuarios. En 2018, gracias a la publicidad de Ling y el acuerdo con la compañía aérea, Brand \& Roses facturó 723.324 euros, el 35\% de los ingresos totales y más del doble de lo que ingresan por las versiones digital e impresa de Yorokobu. Han publicado también la revista Hi! para la cadena hotelera $\mathrm{H} 10$ hoteles, y fueron los responsables de la revista impresa trimestral de Jot down entre 2012 y 2015.

Jot down ha diversificado su negocio con la participación en Soiden, empresa que, además de su revista, distribuye otras como $5 W$, Altaïr o Granta. La distribuidora es una importante fuente de recursos dado que reporta ingresos anuales de en torno a 250.000 euros, el $29 \%$ del total de sus ingresos.

A otro nivel, todos los proyectos han buscado ingresos a través del merchandising, y en algunos casos como en el de Panenka con un $13 \%$ del total ha resultado una vía de ingresos de cierta importancia. O han organizado charlas, talleres, ciclos o participado en conferencias, actividades que, por ejemplo, en el caso de $5 W$, reportan el $3 \%$ de los ingresos.

\subsection{Un sector en fase de crecimiento}

Los proyectos analizados presentan un crecimiento muy importante en cuanto a volumen de negocio. El proyecto de mayor envergadura entre los cinco casos estudiados es el de Brand \& Roses con unos ingresos que se han estabilizado en cifras que superan los dos millones de euros. En la evolución de la facturación anual, desde los 634.000 euros de 2011 a los más de dos millones de euros en 2018, se puede apreciar un crecimiento de un 225\%. Los datos de la evolución de la facturación anual de Jot down muestran un crecimiento de 47,3\% desde los 579.000 euros de 2014 a los 852.000 en 2017. Las facturaciones anuales de CTXT y $5 W$ también han experimentado un crecimiento notable. El volumen de negocio de CTXT ha crecido un 165,5\% desde los casi 200.000 euros de 2016 a los 528.000 euros de 2018, mientras que el de $5 W$ ha aumentado un 126,9\% desde los 104.000 de 2016 a los 236.000 de 2018. De los datos económicos facilitados por los responsables de Panenka se puede deducir que su volumen de negocio rondaba el medio millón de euros en 2017.

Los datos económicos vienen acompañados generalmente de un crecimiento de las audiencias. Ese crecimiento es más moderado en el caso de CTXT que pasó de una media de 593.000 usuarios únicos al mes de 2016 a los 628.000 de 2018 ; fue más pronunciado en los casos de $5 W$ y Panenka, que en el mismo período de tiempo pasaron de 14.850 a 33.152 y de 45.000 a 86.300 , respectivamente. El único que ha tenido una leve bajada en los últimos años ha sido Jot down, que ha pasado de 716.809 a 679.510 usuarios únicos al mes desde el 2016 al 2018. El crecimiento general se refleja también en el número de usuarios de las redes sociales. Yorokobu, por ejemplo, ha pasado de 87.000 seguidores en Facebook en 2013 a 626.000 a comienzos de 2020, o de 61.000 seguidores en Twitter a 114.000 en el mismo período de tiempo.
Los datos indican un crecimiento sostenido en el volumen de negocio, y una evolución generalmente favorable de sus cuentas de resultados, pero siempre dentro de un modelo muy ajustado que condiciona las posibilidades de desarrollo de sus proyectos periodísticos 
Todas las empresas analizadas coinciden en la dificultad para equilibrar su cuenta de resultados anual. Jot down tuvo pequeñas pérdidas en $2014(-7.484$ euros), 2015 (-10.548) y $2016(-8.373)$ y modestas ganancias en 2017 (6.133). Las cuentas de CTXT y $5 W$ arrojaron también resultados muy ajustados. El balance de CTXT evolucionó favorablemente: desde los números negativos de 2015 (-84.336) y 2016 (-29.978) a prácticamente equilibrar las cuentas en 2017 (-2.363), y a obtener ganancias en 2018 (23.214). En cambio $5 W$ ha experimentado una evolución inversa: pasó de reflejar números positivos en 2016 (25.000) y 2017 (10.000) a tener pérdidas en 2018 (-21.400), siempre dentro de una cuenta de resultados ajustada. La responsable de negocio de $5 \mathrm{~W}$ explica las ganancias de los dos primeros años:

"El primer año (...) tuvimos unos ingresos muy fuertes por el crowdfunding. El año pasado también tuvimos ingresos fuertes, nos dieron una beca de Google (...)" (Izcue, 2018).

Los datos relativos a las cuentas económicas de la mayoría de los casos estudiados indican un crecimiento sostenido en el volumen de negocio y una evolución generalmente favorable de sus cuentas de resultados, pero siempre dentro de un modelo muy ajustado que condiciona las posibilidades de desarrollo de sus proyectos periodísticos. Las dificultades económicas a las que se enfrentan los medios slow imponen límites a posibles inversiones para mejorar la calidad de su oferta, así como para diversificarla.
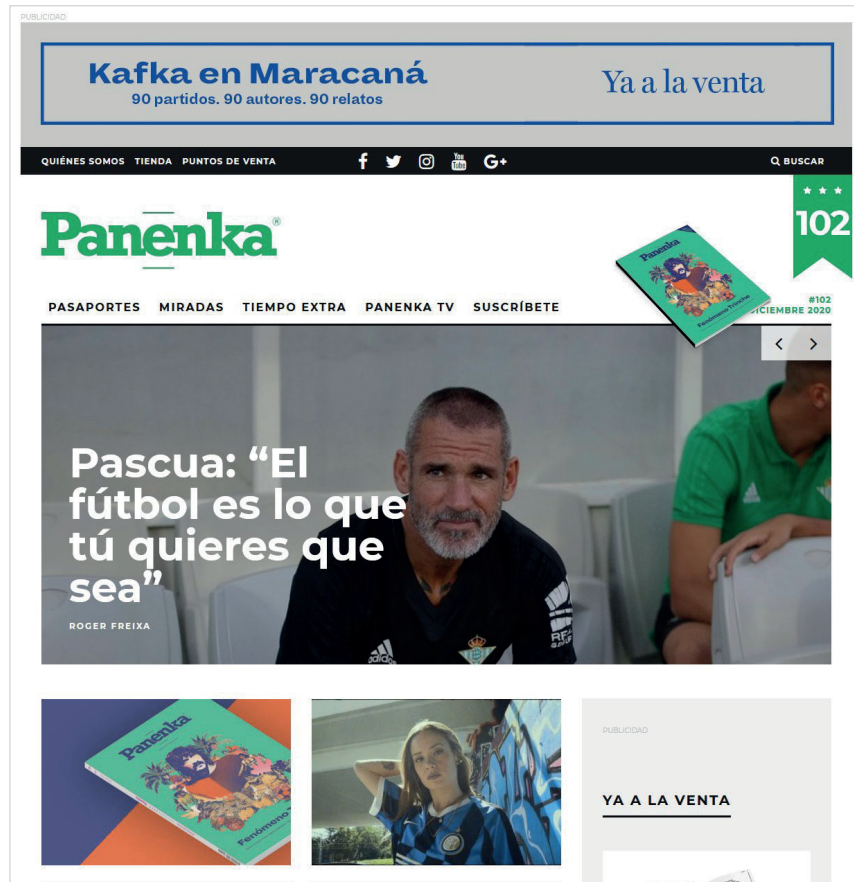

\section{En el}

\#Panenka102, el

fenómeno

Trinche

REDACCIÓN
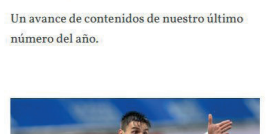

https://www.panenka.org

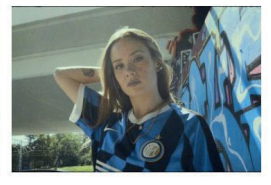

Santa Salut: "Ni ellas van a dejar la pelota ni nosotras el micro" arnau secuna Una charla con la rapera Santa Salut sobre yel papel de las mujeres.

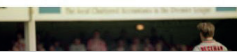

YA A LA VENTA

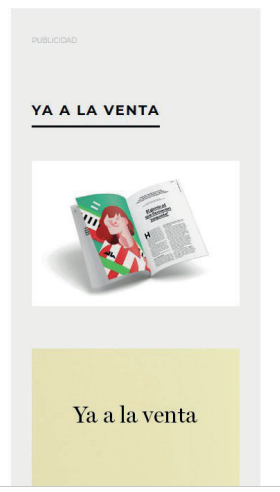

Ya a la venta (1)

Varios de sus responsables apuntan que les gustaría mejorar su oferta audiovisual pero que no pueden afrontar los costes que ello supondría. En Yorokobu producen la mayoría de sus vídeos cuando lo financian las marcas, pero rara vez para la revista (Hurst, 2018); en CTXT sólo como colaboración de algún profesional especializado en el área (Jiménez, 2018); 5W (Morales, 2018) y Jot down (Fernández, 2018) dicen que necesitan más inversión para ser competitivos; y Panenka ha tenido experiencias a nivel audiovisual, mediante colaboraciones con Copa 90, pero se ve demasiado pequeña como para acometer el reto audiovisual como es debido (Xuriach, 2018).

Los expertos del Delphi opinan que la tecnología avanza en dirección al consumo audiovisual en pequeña pantalla y, por tanto, las organizaciones slow deben desarrollar esta vía. Por su parte, los usuarios suspenden a los medios digitales en esta materia: el 40\% de los encuestados en España considera que no se utilizan los recursos audiovisuales como deberían, frente a un $31 \%$ que los ve aceptables y un $29 \%$ que manifiesta no tener una opinión formada al respecto.

Las limitaciones del mercado del periodismo slow son incontestables, pero la evolución de los datos económicos de los casos estudiados, así como las conclusiones de los expertos consultados en la encuesta Delphi, indican que sigue habiendo un segmento de mercado para este tipo de periodismo. Pese a sus limitaciones los promotores de estos proyectos se mantienen firmes en su apuesta por el slow journalism digital. De cara al futuro, en Panenka aspiran a un "crecimiento sostenido" que combine las apuestas por el papel y la web (Beltrán, 2018). En Yorokobu han decidido volver a pausar el ritmo después de haber acelerado la producción:

"La oferta de información en la red es enorme y optamos por reducir el número de entradas y aumentar la calidad de las mismas" (Moreno, 2018).

Los objetivos a corto plazo de Jot down son incrementar hasta un $50 \%$ la comunidad digital y reforzar los contenidos audiovisuales y a medio-largo plazo extender la audiencia entre el público latinoamericano (Fernández, 2018). En 5W aspiran a poder hacer más coberturas propias financiadas por la revista, a practicar más longform elaborando crónicas más extensas y a publicar más contenido audiovisual (Morales, 2018). CTXT tiene como objetivo crecer para poder ofrecer una mayor cantidad de contenido de calidad (Mora, 2018).

De las respuestas del Delphi se concluye que los expertos confían en que habrá un nicho para este tipo de periodismo sostenible, que puede ir creciendo, gracias a dos factores: el mayor nivel educativo de las nuevas generaciones y los niveles de saturación que va alcanzando el periodismo de consumo rápido. 
También la encuesta ofrece datos que invitan al optimismo. Se ha podido constatar que los usuarios de este tipo de periodismo le dan una buena nota: un 3,9 sobre 5, en España. Y, como ya se ha señalado, aunque la cultura de lo gratuito sigue muy enraizada, el pago por contenidos de calidad empieza a abrirse camino entre los lectores de este tipo de prensa, ya que el $54 \%$ de los encuestados se muestra dispuesto a pagar por los contenidos de un periodismo digital de calidad (frente al $27 \%$ de los encuestados que no son lectores de este tipo de prensa).

\section{Conclusiones}

Los cinco proyectos periodísticos estudiados coinciden en su forma jurídica como sociedades limitadas y en un cierto espíritu cooperativista en cuanto a su estructura empresarial. Los socios mayoritarios de todos estos medios son los mismos que fundaron el proyecto, aunque en algunos casos se abre la posibilidad de incorporar a trabajadores a la propiedad de la empresa. Por lo tanto, el control de la empresa queda en manos de los mismos que desarrollan las bases del proyecto periodístico.

Los gastos estructurales son normalmente bastante limitados: los equipos en redacción son mínimos, se trabaja mucho a través de colaboradores, y la mayoría tampoco tiene una redacción física al uso. Es este sentido no trabajan con las pautas de edición de los medios tradicionales: normalmente, funcionan mediante teletrabajo, su forma de producción es ágil y flexible y las previsiones se realizan con tiempos mucho más dilatados.

La comunidad de lectores es central en estos proyectos. Su apoyo económico a través de las suscripciones resulta vital. Aunque todos los proyectos analizados son nativos digitales, no han renunciado a la producción en papel pues constituye una fuente importante de ingresos. No solo la web y el papel actúan complementándose; las redes sociales, aunque no sean una fuente de ingresos en sí, atraen a gran número de lectores.

Algunos medios, convencidos de su rol de intermediario social, ofrecen gratis todo o parte de su contenido digital. Esta decisión tiene una doble vertiente: por un lado favorece el acercamiento de los lectores al medio, pero por otra parte es difícil romper la cadena "internet-igual-a-gratuidad". Y ya se sabe que

"el apelativo de gratuidad es falso, porque es un intercambio puro y duro en el que alguien da algo a cambio de algo" (Campos-Freire, 2010).

Si bien en el Delphi los expertos han mostrado confianza en que los usuarios quieran pagar por los productos de calidad que ofrece el periodismo slow, lo cierto es que las encuestas dicen lo contrario. A nuestro entender, asegurar la fidelidad de los lectores y suscriptores no es tarea fácil: los usuarios buscan información continuamente en la red, y al mismo tiempo van buscando nuevos medios para saciar su sed informativa. La permanencia en "su" medio no está asegurada.

Otra fuente de ingresos relevante es la venta de contenidos, ya sea para que medios más grandes los publiquen como propios (Yorokobu en la revista Ling; Panenka con Copa 90), o para que estos medios más grandes los distribuyan con la marca del proyecto slow (CTXT con Público; Jot down con El país). Y se opta también por el intercambio gratuito de contenidos con medios amigos, como modo de abaratar gastos.

A partir de estas coincidencias cada proyecto ha tenido que encontrar su camino. Yorokobu, la iniciativa que ha conseguido un mayor volumen de negocio de entre los analizados, llega más allá en la venta de contenidos, y, de hecho, el core business de su empresa editora es producir para el marketing de marcas comerciales. Jot down tiene un ingreso importante a través de su participación en una empresa de distribución de libros y revistas. O la venta de merchandising le ha dado buenos resultados a Panenka. En el caso de CTXT y $5 W$ redondean su presupuesto con lo obtenido con talleres, conferencias, becas y premios.

La importancia de la publicidad como fuente de ingresos es relativa. En algunos casos es importante, aunque no llegue a ser la principal fuente de financiación como suele suceder en los medios más grandes. En otros casos esa importancia es más bien escasa e incluso se llega a recelar de esta vía de financiación ya que se considera que puede hacer peligrar la independencia periodística del medio.

Esta diversificación se ha traducido en la falta de un modelo de negocio uniforme. Sin embargo, todos los proyectos han visto crecer su volumen de negocio y sus audiencias. $Y$ ello a pesar de lo ajustado de sus presupuestos, otra característica común de estos medios. Se trata de un sector en crecimiento, sí, pero al que le está costando consolidarse. Estas dificultades limitan en cierta manera el desarrollo de sus proyectos periodísticos, sobre todo para la producción de los contenidos más caros, los audiovisuales.
De las respuestas del Delphi se concluye que los expertos confían en que habrá un nicho para este tipo de periodismo sostenible, que puede ir creciendo, gracias a dos factores: el mayor nivel educativo de las nuevas generaciones y los niveles de saturación que va alcanzando el periodismo de consumo rápido 
Algunos participantes del Delphi han destacado que la búsqueda de la independencia de los proyectos slow es central para afianzarse económicamente. La piedra angular, entonces, es cómo materializar y salvaguardar la independencia editorial de estos medios que en general no son muy rentables.

\section{Bibliografía}

$5 W$

https://www.revista5w.com

Abad, Mar (2018). Entrevista personal, Madrid, 16-04-2018.

Abella, Fermin (2018). Entrevista personal, Bilbao, 22-03-2018.

Arias, Marta (2018). Entrevista personal, Barcelona, 16-03-2018.

Asociación de la Prensa de Madrid (2014). “Otro escenario". Informe anual de la profesión periodística 2015. Madrid: APM, pp. 92-101.

https://www.apmadrid.es/wp-content/uploads/2009/02/Informe\%20profesion_2014_def_baja.pdf

Asociación de la Prensa de Madrid (2018). "Los medios siguen sumidos en la incertidumbre". Informe anual de la profesión periodística 2018. Madrid: APM, pp. 64-87.

https://www.apmadrid.es/wp-content/uploads/2019/07/Informe-profesi\%C3\%B3n-2018_baja.pdf

Beltrán, Marcel (2018). Entrevista personal, Barcelona, 09-07-2018.

Briggs, Mark (2012). Entrepreneurial journalism: How to build what's next for news. Los Angeles-London: Sage. ISBN: 9781608714209

Campos-Freire, Francisco (2010). "Las empresas de medios de comunicación revisan y amplían sus modelos de negocio". Razón y palabra, v. 74.

http://www.razonypalabra.org.mx/N/N74/VARIA74/16FreireV74.pdf

Casero-Ripollés, Andreu (2010). "Prensa en internet: nuevos modelos de negocio en el escenario de la convergencia”. EI profesional de la información, v. 19, n. 6, pp. 595-601.

https://doi.org/10.3145/epi.2010.nov.05

Casero-Ripollés, Andreu; Cullell-March, Cristina (2013). “Periodismo emprendedor. Estrategias para incentivar el autoempleo periodístico como modelo de negocio". Estudios sobre el mensaje periodístico, v. 19, pp. 681-690.

https://doi.org/10.5209/rev_ESMP.2013.v19.42151

Cea-Esteruelas, María-Nereida (2013). "Economía de los cibermedios: modelos de ingresos y fuentes de financiación". El profesional de la información, v. 22, n. 4, pp. 353-361.

https://doi.org/10.3145/epi.2013.jul.12

CTXT

https://ctxt.es

Dowling, David (2016). "The business of slow journalism". Digital journalism, v. 4, n. 4, pp. 530-546.

https://doi.org/10.1080/21670811.2015.1111769

Drok, Niko; Hermans, Liesbeth (2016). “Is there a future for slow journalism?”. Journalism practice, v. 10, n. 4, pp. $539-554$. https://doi.org/10.1080/17512786.2015.1102604

Fernández, Ángel-Luis (2018). Entrevista personal, Sevilla, 15-03-2018.

García-Avilés, José-Alberto; González-Esteban, José-Luis (2012). “Cibermedios nativos españoles: explorando modelos de rentabilidad". Tripodos, n. 30, pp. 153-167.

http://www.tripodos.com/index.php/Facultat_Comunicacio_Blanquerna/article/view/50/360

García-Galindo, Juan-Antonio; Cuartero-Naranjo, Antonio (2015). “Le journalisme narratif en espagnol dans la société de l'information". Communication, v. 33, n. 2.

http://communication.revues.org/5823

Gómez-Mompart, Josep-Lluís; Gutiérrez-Lozano, Juan-Francisco; Palau-Sampio, Dolors (2015). “Los periodistas españoles y la pérdida de calidad de la información: el juicio profesional”. Comunicar v. 23, n. 45, pp. 143-150.

https://doi.org/10.3916/C45-2015-15

Greenberg, Susan (2007). "Slow journalism”. Prospect, 25 de febrero. https://www.prospectmagazine.co.uk/magazine/slowjournalism

Greenberg, Susan (2012). "Slow journalism in the digital fast lane”. In: Lance, Richard; Tulloch, John (eds.). Global literary journalism: exploring the journalistic imagination. Berna: Peter Lang Inc., International Academic Publishers, pp. 381393. ISBN: 9781433118661 
Honoré, Carl (2012). Elogio de la lentitud. Un movimiento mundial desafía el culto a la velocidad. Barcelona: RBA. ISBN: 9788478715282

Hurst, Marcus (2018). Entrevista personal, Madrid, 16-04-2018.

Izcue, Maribel (2018). Entrevista personal, Barcelona, 15-03-2018.

Jiménez, Vanesa (2018). Entrevista personal, Madrid, 25-05-2018.

Jot Down

https://www.jotdown.es

Juntunen, Laura (2010). "Explaining the need for speed: speed and competition as challenges to journalism ethics". In: S. Cushion \& J. Lewis (eds.). The rise of 24-hour news television: global perspectives. New York: Peter Lang, pp. 167-181. ISBN: 9781433107764

Kent, Michel; Saffer, Adam (2014). A Delphi study of the future of new technology research in public relations. Public relations review, v. 40, n. 3, pp. 568-576.

https://doi.org/10.1016/j.pubrev.2014.02.008

Köhler, Benedikt; David, Sabria; Blumtritt, Jörg (2010). The slow media manifesto. http://en.slow-media.net/manifesto

Landeta, Jon (1999). El método Delphi: una técnica de previsión para la incertidumbre. Barcelona: Arial. ISBN: 84344 28369

Le-Masurier, Megan (2015). "What is slow journalism". Journalism practice, v. 9, n. 2, pp. 138-152.

https://doi.org/10.1080/17512786.2014.916471

Lindlof, Thomas; Taylor, Bryan (2011). Qualitative communication research methods. (3 ${ }^{\text {rd }}$ ed.). Los Angeles: Sage. ISBN: 9781412974738

Linstone, Harold; Turoff, Murrai (2002). The Delphi method: Techniques and applications (18). Boston: Addison-Wesley Educational Publishers Inc. ISBN: 9780201042931

Manfredi-Sánchez, Juan-Luis; Rojas-Torrijos, José-Luis; Herranz-de-la-Casa, José-María (2015). “Innovación en el periodismo emprendedor deportivo. Modelo de negocio y narrativas". El profesional de la información, v. 24, n. 3, pp. 265-273.

https://doi.org/10.3145/epi.2015.may.06

Manias-Muñoz, Itsaso; Jin, Yan; Reber, Bryan H. (2019). "The state of crisis communication research and education through the lens of crisis scholars: An international Delphi study". Public relations review, v. 45, n. 4, 101797. https://doi.org/10.1016/j.pubrev.2019.101797

Marín, Alba (2016). "Periodismo emergente: Atención al usuario, calidad e hibridación. Estudio del caso Revista $5 W$ en España”. En: Moreno-Espinosa, Pastora (coord.). Comunicación, ciberperiodismo y nuevos formatos multimedias interactivos, pp. 32-47. Sevilla: Ediciones Egregius. ISBN: 9788494524370

https://idus.us.es/xmlui/bitstream/handle/11441/48546/Pages\%20from\%20ciberperiodismoynuevos-3.pdf

Martín-Río, Carlos (2018). Entrevista personal, Barcelona, 09-07-2018.

Mora, Miguel (2018). Entrevista personal, Madrid, 24-05-2018.

Morales, Agus (2018). Entrevista personal, Barcelona, 15-03-2018.

Naudín-Escuder, Eduardo (2018). "Nuevas narrativas: Análisis del periodismo millenial en los medios españoles". En: Rodríguez-Rodríguez, J. M.; López-Pan, F.; Albalad Aiguabella, J. M. (coords.). Calidad informativa y nuevas narrativas. Zaragoza: Egregius, pp. 41-68. ISBN: 9788417270469

https://egregius.es/catalogo/calidad-informativa-y-nuevas-narrativas

Neveu, Erick (2014). "Revisiting narrative journalism as one of the futures of journalism”. Journalism practice, v. 15, n. 5, pp. 533-542.

https://doi.org/10.1080/1461670X.2014.885683

Neveu, Erick (2016). "On not going too fast with slow journalism”. Journalism practice, v. 10, n. 4, pp. 448-460. https://doi.org/10.1080/17512786.2015.1114897

Newman, Nic (2016). Media, journalism and technology predictions 2016. Oxford: Reuters Institute for the Study of Journalism.

https://reutersinstitute.politics.ox.ac.uk/sites/default/files/2017-06/Journalism\%2C\%20media\%20and\%20technology\%20 predictions\%202016.pdf 
Palau-Sampio, Dolors; Cuartero-Naranjo, Antonio (2018). “El periodismo narrativo español y latinoamericano: influencias, temáticas, publicaciones y puntos de vista de una generación de autores". Revista latina de comunicación social, v. 73, pp. 961-979.

http://doi.org/10.4185/RLCS-2018-1291

Panenka

https://www.panenka.org

Ramírez-de-la-Piscina, Txema; Aiestaran, Alazne; Agirre, Antxoka; Zabalondo, Beatriz (2015). Quality journalism: Is there a future? The evolution of the reference press. Leioa: UPV/EHU. ISBN: 9788490822166

Rauch, Jennifer (2011). "The origin of slow media: Early diffusion of a cultural innovation through popular and press discourse, 2002-2010". Transformations, n. 20.

http://www.transformationsjournal.org/wp-content/uploads/2016/12/Rauch_Trans20.pdf

Rodríguez-Rodríguez, Jorge-Miguel; Albalad-Aiguabella, José-María (2012). “Nuevas ventanas del periodismo narrativo en español: del big bang del boom a los modelos editoriales emergentes". Textual \& visual media, n. 5, pp. $287-310$.

http://www.textualvisualmedia.com/es/archivo/14-archivo-de-revistas/36-revista- $n-6$

Rojas-Torrijos, José-Luis; Marín-Sanchiz, Cristian-Ramón (2016). “Modelos de negocio para el periodismo deportivo de nicho en el contexto postindustrial". Ámbitos, n. 33.

http://institucional.us.es/ambitos/?p=2484

Rosenberg, Howard; Feldman, Charles (2008). No time to think. The menace of media speed and the 24-hour news cycle. New York: Continuum. ISBN: 9780826429315

Rosique-Cedillo, Gloria (2012). “Comunicación e información slow en la era digital: La utopía posible”. En: Victoria-Mas, Juan-Salvador; Gómez-Tinoco, Alicia; Arjona-Martín, José-Borja (coords.). Comunicación slow (y la publicidad como excusa). Madrid: Fragua, pp. 315-342. ISBN: 9788470745195

Rosique-Cedillo, Gloria; Barranquero-Carretero, Alejandro (2015). “Periodismo lento (slow journalism) en la era de la inmediatez. Experiencias en Iberoamérica". El profesional de la información, v. 24, n. 4, pp. 451-462.

https://doi.org/10.3145/epi.2015.jul.12

Sabaté-Gauxachs, Alba; Micó-Sanz, Josep-Lluís; Díez-Bosch, Miriam (2019). "Is the new new digital journalism a type of activism? An analysis of Jot down, Gatopardo and The New Yorker". Communication \& society, v. 32, n. 4, pp. $173-191$. https://doi.org/10.15581/003.32.4.173-191

Saltzis, Kostas (2012). "Breaking news online. How news stories are updated and maintained around-the-clock". Journalism practice, v. 6, n. 5-6, pp. 702-710.

https://doi.org/10.1080/17512786.2012.667274

Spark, David (2012). Investigative reporting. A study in technique. New York: Routledge. E-book. ISBN: 9780080509396

Taborda, Luciana-Agustina (2016). El crowdfunding y el periodismo lento en medios de comunicación digital. Trabajo fin de master. Badajoz: Universidad de Extremadura.

http://dehesa.unex.es/handle/10662/5017

Vara-Miguel, Alfonso; Díaz-Espina, Carolina (2012). "Modelos de negocio y estrategia editorial: el caso del wsj.com". Revista de comunicación, n. 11, pp. 110-128.

https://dadun.unav.edu/bitstream/10171/27752/1/WSJ\%20publicado.pdf

Wimmer, Roger; Dominik, Joseph (1996). La investigación científica de los medios de comunicación. Una Introducción a sus métodos. Barcelona: Bosch. ISBN: 9788476763599

Wirtz, Bernd W. (2011). Media and internet management. Wiesbaden: Gabler Verlag. ISBN: 9783834930101

Xuriach, Roger (2018). Entrevista personal, Barcelona, 09-07-2018.

Yin, Robert (1992). "The case study method as a tool for doing evaluation”. Current sociology, v. 40, n. 1, pp. $121-137$. https://doi.org/10.1177/001139292040001009

Yorokobu

https://www.yorokobu.es

Yuste, Bárbara; Cabrera, Margarita (2014). Emprender en periodismo. Nuevas oportunidades para el profesional de la información. Barcelona: Editorial UOC. ISBN: 9788490640708 